\title{
La mesure précise et l'enregistrement des niveaux stables ou fluctuants au moyen de pointes limnimétriques à vibrations entretenues
}

\section{The accurate measuring and the recording of stable or fluctuating water-levels by means of continually vibrating limnimetric points}

\author{
PAR H. GRIDEL
}

INGÉNHEUR-DOCTEUR

Martre de Compinexces a I'Fcole Centrale des Arts et Manupactures

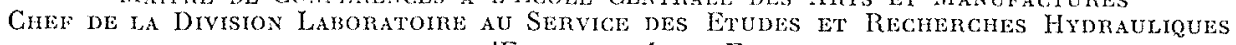
de x'Electricité de France

English synopsis p. 637

\section{Préambule}

Un des chapitres les plus importants de la Métrologie, en matière de Mécanique des Fluides, est celui qui concerne la détermination, absolue ou relative, discontinue ou permanente, de la position des surfaces libres des liquides.

$\mathrm{Si}$ ces mesures sont en effet le but direct recherché pour les relevés de lignes d'eau des écoulements à veine libre, pour l'étude quantitative des remous, des ressauts, la détermination des pertes de charge de ces écoulements, etc..., elles intéressent également, par le truchement d'appareils de mesures classiques, les relevés de pressions qui, du fait de l'utilisation de manomètres à colonnes liquides, se ramènent à des mesures de dénivellation entre les deux branches du manomètre; au même titre, les mesures de vitesse, ramenées à des mesures différentielles de pression quand on utilise des tubes de Pitot et des appareils déprimogènes sont tributaires de la connaissance relative de deux niveaux.

D'où la grande variété des méthodes de me- sures imaginées pour s'appliquer au mieux aux divers types d'opérations à effectuer.

Parmi celles-ci, spécialement pour les mesures précises, celle qui utilise la pointe limnimétrique est trop comnue pour qu'il soit nécessaire d'en décrire ici les détails : une règle graduée, pourvue à sa partie inférieure d'une pointe que l'on amène au contact du liquide, permet d'en apprécier le niveau par la simple lecture de la graduation de la règle, celle-ci se trouvant en face d'un repère fixe dont le nivellement a été effectué à l'avance une fois pour toutes; l'avantage de cette méthode, ontre sa simplicité, réside dans le fait que seule l'extrémité de la pointe est en contact avec le liquuide dont, ainsi, l'écoulement ne se trouve pas perturbé par le dispositif de mesurc.

Le développement actuel de la technique des essais sur modèles réduits, comme la poursuite, dans les mesures industrielles, de précisions toujours pluss grandes, conduit à rechercher dans toutes les limites du possible les perfectionnements que l'on peut apporter à cette technique de mesure aussi bien dans le scns de la préci- 
sion que dans celui de la commodité d'emploi.

C'est ainsi que, pour améliorer la précision de la méthode et réduire la fatigue des expérimentateurs, on remplace maintenant de facon courante la détermination visuele du contact de la pointe par un procédé électrique de détection (qui évite les erreurs dues à l'équation personnelle de l'observateur : une lampe à néon ou mieux un trèfle cathodique s'éclaire brusquement quand la pointe, arrivant au contact avec l'eau, ferme un circuit électrique dans lequel il est inséré : on peut même se donner une fourchette de précision en jumelant deux pointes légèrement décalíes en hauteur qui actionnent successivement le trille cathodique dans la bande d'erreur que l'on s'est fixée a priori lors du réglage des pointes.

Si le circuit est asse\% rósistant pour que l'ean puisse ètre considéréc comme un bon conducteur de l'électricité, le degré d'enfoncement de la pointe dans l'cau n'intervient absolument pas sur l'intensité qui traverse le dispositif détecteur et par conséquent, la position pour laquelle la pointe touche l'eau est déterminée sans ambiguité de même que l'instant où, dans le mouvement descendant de la pointe, le contact vient à s'établir.

En général, le dispositif porte-pointe comporte un vernier au $1 / 10^{\circ}$ et la règle est graduée en mm. Dans ce cas, la précision de la lecture concorde avec la précision de l'équipement et on peut mesurer la position d'un plan d'eau stable an $1 / 10^{\circ}$ de $\mathrm{mm}$ près : an contraire, avec le simple contrôle visuel du contact de la pointe, Je $1 / 4$ de $\mathrm{mm}$ n'est atteint avec certitude que par des expérimentateurs très méticuleux: la précision qu'implique la présence du vernier n'est alors qu'apparente.

Au reste, même avec une détection électrique, il serait illusoire de rechercher une précision plus grande, même pour des mesures statiques, par une amélioration du vernier, car on serait alors limité par les effets de tension superficielle du fluide: au moment du contact, la pointe mouille, et la surface de l'eau remonte sur la tige en formant une colonne en forme de toit de pagode qui s'élève au-dessus de la surface horizontale initiale du fluide d'une quantité mal précisée; la détection même électrique du contact, perd alors toute signification du fait de la tension superficielle de l'eau.

D'autre part, lorsqu'on releve fortement la pointe, la colonne liquide s'allonge, puis, après rupture, laisse une goutte au bout du limnimètre dont il faut attendre la chute pour recommencer la mesure : on concoit que, si le plan d'eau fluctue quelque peu, les mesures de niveaux perdent alors une bonne part de la précision attendue.

\section{Pointe suiveuse à oscillations de relaxation lentes}

Pour obvier à cet inconvénient et permettre de relever des fluctuations lentes de niveau (marées, ondes de crues, houles en modèle) on a récemment proposé divers dispositifs, tous plus ou moins hasés sur l'emploi d'oscillations périodiques lentes avec effets de relaxation.

Dans un de ces appareils, de réalisation francaise, la pointe, initialement hors d'eau, est fixéc par un fil au treuil d'un petit moteur électrique: lorsque le treuil dévire, sous l'action du poids de l'équipage mobile, la pointe tombe; au contact de l'eau, le circuit se ferme sur un relai ćlectronique sans inertie qui, d'une part, pointe la position du treuil, donc enregistre le niveau iet, d'autre part, met le courant sur le moteur pour un temps limité.

De ce fait, la pointe remonte hor's de l'eau et le cycle peut recommencer dès que le moteur n'est plus alimenté.

Par ce dispositif, on peut obtenir au maximum deux relevés par seconde avec une précision qui, au mieux, est le $1 / 10^{\circ}$ de $\mathrm{mm}$ : en outre, la pointe ne peut guère suivre des fluctuations verticales de niveau supérieures à $2 \mathrm{~cm} / \mathrm{s}$.

Les enregistrements obtenus sont tracés point par point et on ne pent espérer en resserrer la trame car la tension superficielle provoque, sur la pointe, des gouttes que l'on doit laisser retomber, ce qui limite naturellement les cadences possibles de l'oscillation de relaxation.

Au Laboratoire de Delft en Hollande, on a réalisé un dispositif analogue composé essentiellement de deux pointes conductrices suspendues aux deux extrémités d'un fléau, oscillant suivant une loi pendulaire, autour de son axe central. Lorsqu'une pointe, dans son mouvement de descente, touche l'eau, elle agit sur un relai électronique qui pointe sa position et provoque sa remontée, done la descente de l'autre pointe; le mouvement du fléau s'entretient ainsi comme celui d'un balancier d'horloge; en réglant l'inertie du fléau on se tronve maître de la cadence des pointes micux qu'avec un simple dispositif à relaxation, mais on n'en annule pas pour autant les effets de la tension superficielle, ce qui fait que la précision des mesures n'est pas plus grande.

Aux U.S.A. on utilise dans ce même ordre d'idée une pointe à double contact d'un caractère à peine différent.

Une tête isolante mobile verticalement et fixée sur un petit treuil clectrique porte trois fines tiges conductrices, l'une, la plus longue, pour le retour du courant, les deux autres réglées de façon identique à $1 / 4 \mathrm{~mm}$ près. Un schéma électrique approprié fait monter le treuil quand les 
deux pointes détectrices sont dans leau, le fait descendre quand elles sont toutes deux hors d'eau et en provoque l'arrêt lor'squ'une pointe est dans l'cau mais l'autre pas.

On concoit qu'on puisse parvenir ainsi à suivre par paliers successifs de l'ordre du $1 / 4$ de mm les fluctuations d'une surface; cependant, l'expérience prouve que la précision et la rapidité de róponse de ces pointes laissent fortement à désirer.

\section{Pointe suiveuse à oscillations entretenues}

La nécessité d'obtenir, sur des modèles en cours d'ctude au Laboratoire de Chatou, des performances meilleures, nous a conduit à imaginer et à mettre au point un dispositıf nouveau basé sur les remarques suivantes:

- Si l'on imprime à une aiguille verticale conductrice du courant une vibration longitudinale rapide telle que la surface de l'eau se trouve dans les limites de l'oscillation de son extrémité :

1) Quand le circuit électrique de détection du contact entre l'aiguille et l'eau est bien réalisé, l'intensité du comrant qui le traverse ne varie pas tant que la pointe est dans l'eau; on peut donc utiliser, pour caractériser to niveau de l'eau pour chaque oscillation de la pointe, le rapport du temps pendant lequel elle fait contact avec l'eau au temps total de l'oscillation.

2) Pour des fréquences musicales courantes. le contact d'une pointe fine avec l'ean provoque des rides capillaires en phase avee le dispositif vibrant: le niveau relatif de l'eau n'est done plus affecté par le mon:vement de l'aiguille et, dans ce cas, -.. un examen au stroboscope le montre il n'y a plus de goutte liquide adherente à la pointe: dans ces conditions, on peut dire que l'effet de la tension superficiclle se trouve annulé.

\section{Principe et réalisation}

Pour se placer dans ces conditions théoriques, on réalise l'appareil suivant:

Un moteur de haut-parleur magnéto-dynamique privé de son pavillon et de son châssis et muni d'un dispositif spécial de centrage est excité par un courant à fréquence musicale et vibre de facon régulière en période et amplitude.

Sur ce moteur, on fixe une pointe fine qui assure la fermeture d'un circuit très résistant avec l'eau.
Tant que la pointe est dans l'eau, elle est traversée par un courant constant : dès qu'elle est hors d'eau it ne passe plus de courant, on a donc un graphique du courant modulé qui a lallure de la figure 1.

Suivant que la surface libre occupe les positions $a, b$ ou $c$ par rapport à la vibration de la pointe, ie graphique du courant modulé affecte les allures $A, B$ ou $C$ indiquées sur la figure, les aires hachurées représentant les quantités de courant parcourant le circuit pendant le temps de référence considéré.

De ceci il résulte que le courant parcourant le circuit de détection est une fonction simple et
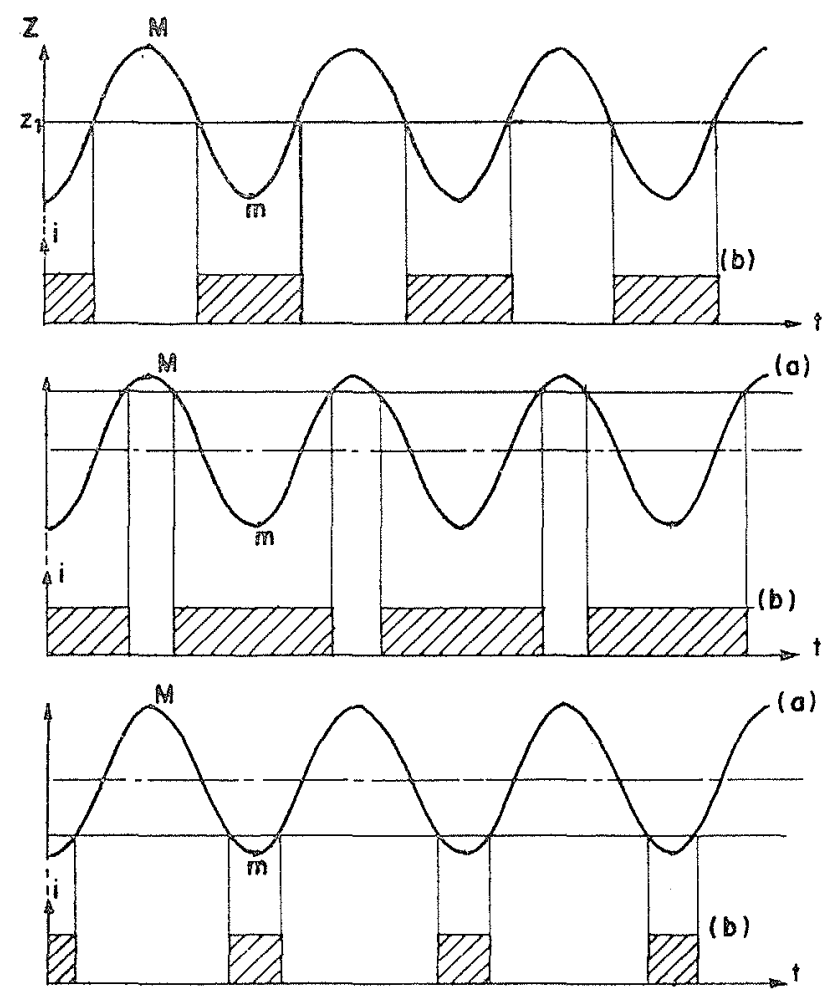

Fiti. 1 .

directe de la position moyenne de la pointe par. rapport à la surface du liquide.

En intégrant ce courant au moyen d'un condensateur et en le recevant sur un oscillographe ou un miero-ampèremètre (dans ce dernier cas le condensateur n’est plus nécessaire car l'inertie de l'équipage mobile de l'ampèremètre tient lieu d'intégrateur) on peut, bien entendu dans le domaine de l'amplitude de la vibration, déceler facilement de très faibles fluctuations de niveau. L'expérience nous a montré que sans prendre de précautions exceptionnelles, la précision dans l'appréciation des fluctuations de niveau pouvait atteindre le $1 / 200^{\circ}$ de $\mathrm{mm}$. 
En outre, le dispositif de détection pouvant ètre sans inertie dans le cas de l'oscillographe par exemple, les fluctuations de niveau les plus rapides sont immédiatement décelables dès que leur fréquence est de l'ordre de grandem de la fréquence musicale excitant la vibration entretenue.

A vrai dire, quelques précautions pritiques

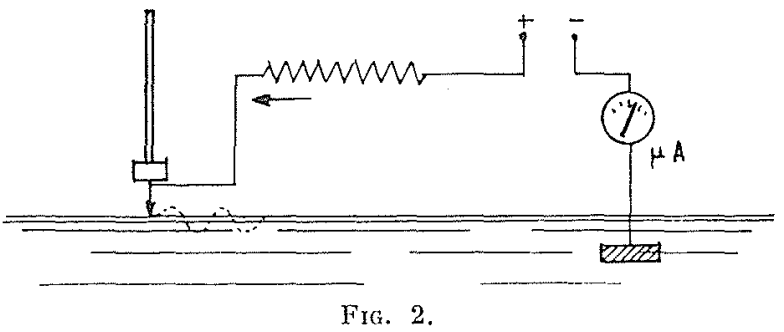

doivent être prises : en effet, le circuiś de létection étant alimenté en courant continu, on constate que si l'on met le + sur la pointe, l'électrolyse de l'eau y dépose des anions, fait apparaitre de l'oxygène naissant d'où oxydation et

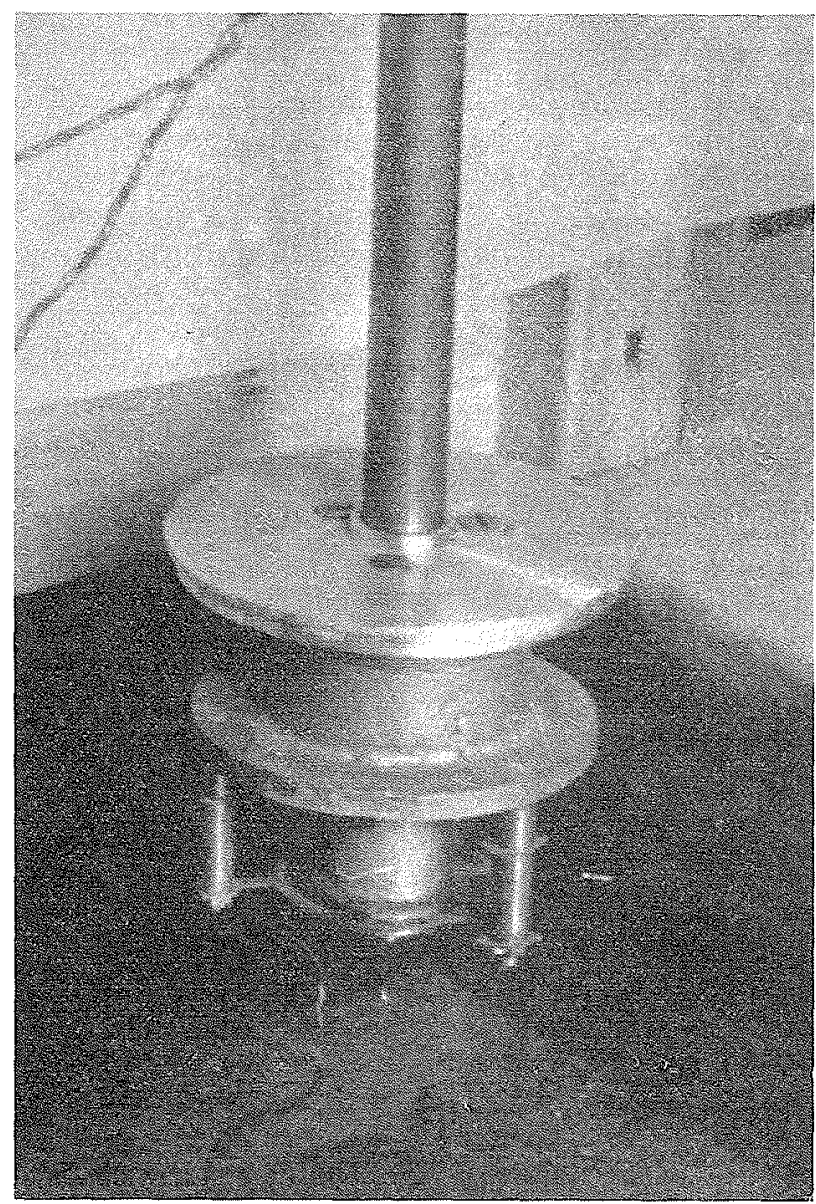

Fig. 3.

Parlie vibrante de l'appareil, le capot de protection enlevé. par là même usure rapide de la pointe; si, au contraire, on y met le --., les cathions déposent sur le fil toutes les impuretés de la surface liquide, ce qui réduit la précision des mesures dans le temps.

Finalement, le problème est résolu simplement. en constituant la pointe par un fil fin de platine sur lequel on met le pôle + du circuit (fig. 2 ).

La figure 3 représente la tête vibrante d'un de nos appareils.

L'inconvénient du schéma qui vient d'être décrit est que la fluctuation de niveau mesurable est limitée à l'amplitude de la vibration du moteur de haut-parleur (un centimètre au maximum pour un moteur courant du commerce) et que dans ce domaine la réponse est loin d'être linéaire dans les zones extrêmes.

On a supprimé ces imperfections en recourant au montage électrique ci-après.

\section{Pointe suiveuse vibrante}

Le mouvement vertical de la tête vibrante est commandé par un petit moteur spécial à conrant continu à excitation séparée et à réponse ultra-rapide. Ce moteur est actionné directement dans un sens ou dans l'autre par un amplificateur relais concu de telle facon que, lorscue la pointe est dans l'eau, un courant bien déterminé passe dans le moteur dans un sens, et quand la pointe est hors d'eau, le même courant passe dans le moteur dans l'autre sens. Le moteur est donc parcouru par un courant tantôt positif, tantôt négatif, et il ne se met en rotation que si les temps de passage de ces deux courants sont différents. Si le moteur est branché dans le bon sens, on concoit qu'il déplacera la tête vibrante par rapport au niveau de l'eau de façon que l'aiguille soit constamment un temps égal dans l'eau et hors d'eau. Le mouvement vertical de la tête reproduira donc, dans les limites de la loi de réponse de l'ensemble, le mouvement du niveau de l'eau.

Bien qu'aucun «pompage » dans le fonctionnement de l'appareil n'ait été observé pendant sa mise au point, ce qui s'explique par le fait qu'il $y$ a pratiquement cinquante points de mesure par seconde, chiffre hors de proportion avec l'inertie du moteur cui, ainsi, moyenne ces mesures, on a par précaution installé dans le moteur un enroulement tachymétrique qui assurera la stabilisation du groupe vibreur même si la loi de montée imposée au groupe venait à être très grande et la vibration de la pointe très petite.

Il va de soi que la précision de la mesure va dans le sens contraire do la limite extrême que l'on s'est imposée pour la loi de mouvement. En 


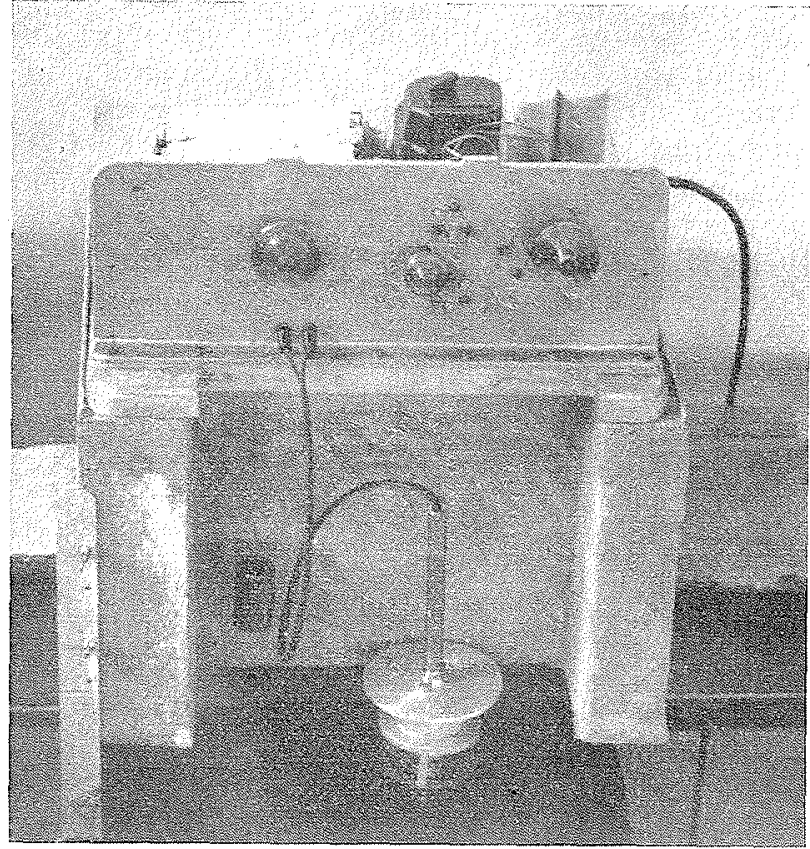

Fili, 1 .

ce qui nous concerne, nous avons mis au point un appareil dans lequel la pointe vibre à 50 périodes par seconde et qui utilise un moteur spécial à réponse très rapide. Nous avons pu obtenir une précision de $1 / 10 \mathrm{~mm}$ avec une vitesse maximum de montée de $5 \mathrm{~cm} / \mathrm{s}$.

Ces résultats nous semblent concilier au mieux les conditions de précision nécessaire et les besoins courants d'un Laboratoire d'hydraulique,

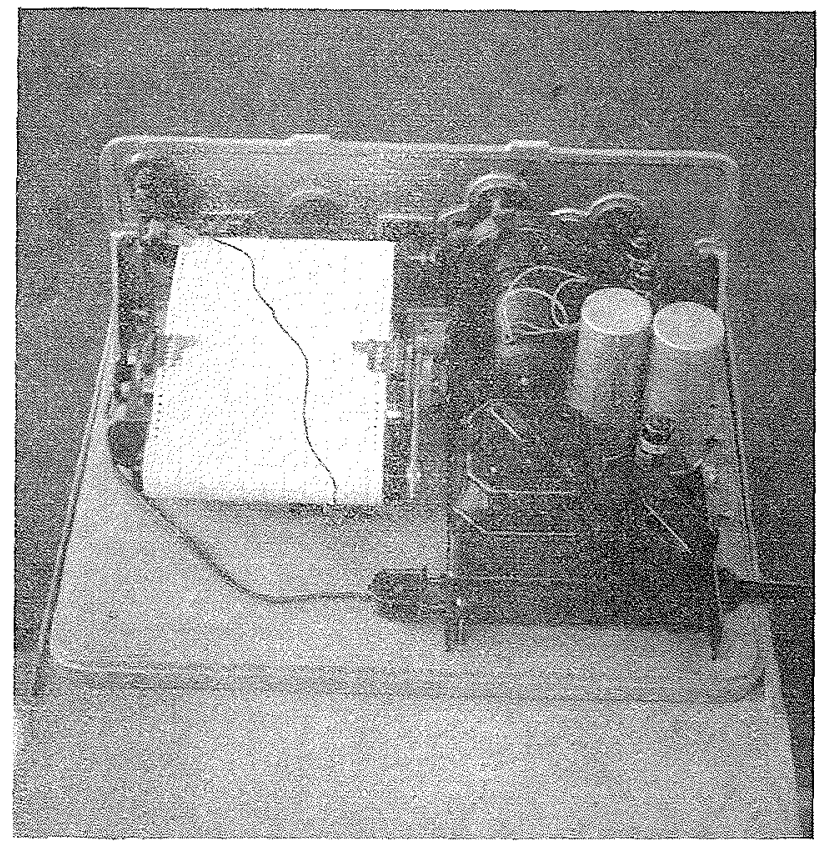

Iili. 5 . mais, bien entendu, l'appareil peut facilement s'adapter à des conditions plus sévères sur la précision si l'on se montre moins exigeant sur la vitesse de montée, et réciproquement.

I.a loi de réponse est telle que l'on peut suivre des fréquences de 1 à 2 . Pour les variations plus rapides, nous étudions actuellement un appareil qui permettra, au moyen d'un milliampèremètre enregistreur spécial, de suivre des variations de niveau jusqu'à des fréquences de 5 à 10 .

D'autre part, le montage est très robuste et ne se dérègle pas : nous avons fait des essais de fonctionnement continu pendant trois mois consécutifs sans aroir le moindre incident de marche.

Les figures 4 et 5 donnent une idée des dispositions constructives adoptées pour la réalisation de l'appareil enregistreur : on doit noter qu'il ne peut fonctionner que si le liquide sur lequel on opère est quelque peu conducteur; s’il n'en était pas ainsi on peut tourner la difficulté en disposant à la surface du liquide une coupelle remplie d'eau portée par un flotteur, donc liée à la loi de niveau à mesurer; cependant la solution est moins simple et la période de réponse s'en ressent.

Par ailleurs, si le licuide conducteur est surmonté d'un liquide isolant tel que l'huile, on a observé que le fonctionnement de l'appareil reste excellent; seulement il indique la position de l'interface: cette propriété est intéressante pour les essais où on veut étouffer les rides capillaires par le recouvrement de la surface au moyen d'huile monomoléculaire.

\section{Appareils différentiels}

Le dispositif s'adapte très bien aux mesures différentielles précises.

Il suffit par exemple (fig. 6) que le moteur $\mathrm{M}_{1}$, commandé par une pointe $P_{1}$ par l'intermédiaire de l'ampli $A_{1}$ entraîne par ailleurs à la même vitesse verticale la pointe $P_{2}$ tandis que $P_{2}$ agit de son côté sur un moteur $\mathrm{M}_{2}$ par l'ampli $\mathrm{A}_{2}$. Dès lors, il est clair que $\mathrm{M}_{1}$ enregistrera les mouvements de $P_{1}$ et $M_{2}$ ceux de $P_{2}-P_{1}$.

Pour des mesures en des points peu éloignés, sur un Venturi par cxemple, nous avons réalisé un montage basé sur le même principe mais d'une réalisation quelque peu différente (fig. 7 ). Le moteur $M$ commandé par la pointe $P_{1}$ au moyen de l'ampli $A$ entraîne un châssis rigide C sur lequel $P_{2}$ est monté par l'intermédiaire d'un palmer. Le circuit de $\mathrm{P}_{2}$ est relié à un microampèremètre A; bien entendu, les niveaux à comparer sont ramenés dans des pots de mesure très voisins, de manière à diminuer les dimensions, donc les erreurs provenant de déforma- 


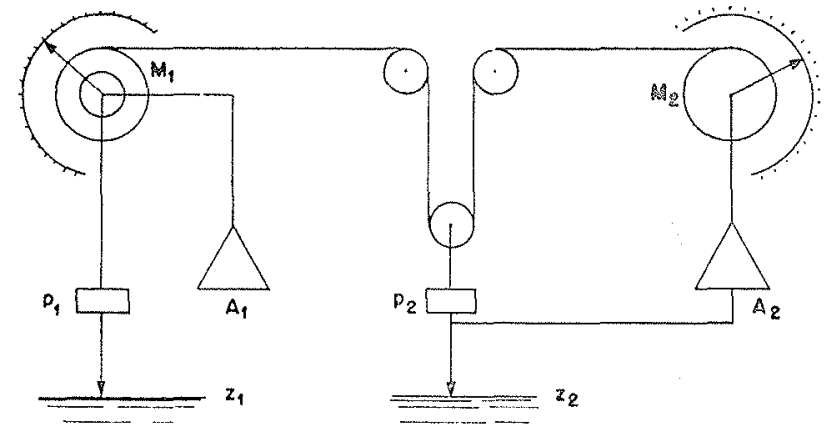

FIC. 6.

tions du châssis $C$. Si les deux pointes sont de niveau au début d'un essai, le micro-ampiremètre étant au zéro, on peut, quand une diffé-

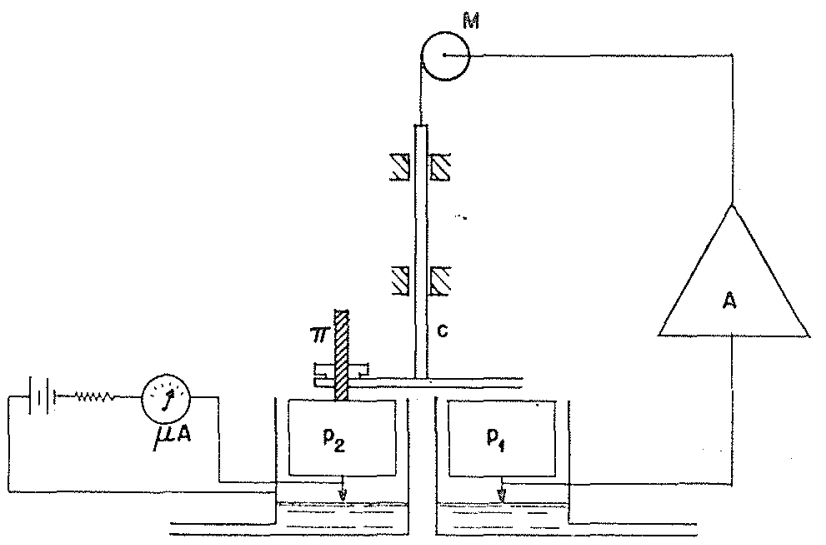

FIG: 7 . rence de niveau s'établit, la compenser par le palmer jusquà ce que le micro-ampiremètre soit de nouveau revenu au zéro. On lit alors la différence sur le palmer avec la précision de mesure que donne le palmer d'une part of la méthode du zéro d'autre part; on peut également ne pas toucher au palmer et étalonner préalablement le miero-ampèremètre en dénivellation que l'on lira ensuite directement. Dans l'un ou l'autre cas, les lectures au $1 / 100^{\circ}$ de millimitre sont très aisées, surtout si les variations de niveaux ne sont pas trop rapides.

\section{Conclusion}

Réalisé, mis au point et essayé depuis plus d'un an au Laboratoire National d'Hydraulique de Chaton, l'appareil que nous venons de décrire. a rendu déjà de très grands services pour la détermination des lignes d'eau et la mesure de très petites pertes de charge.

Du fait de ses performances qui sont sensiblement plus étendues que celles des appareils antérieurs, la pointe vibrante permettra de réaliser des modiles plus petits, toutes les fois que la précision demandée des mesures conditionne l'échelle de réduction des maquettes. La figure 8 représente un enregistrement de houle dans un modèle au $1 / 300^{\circ}$.

Par ailleurs, la faculté de mesurer avec pré-

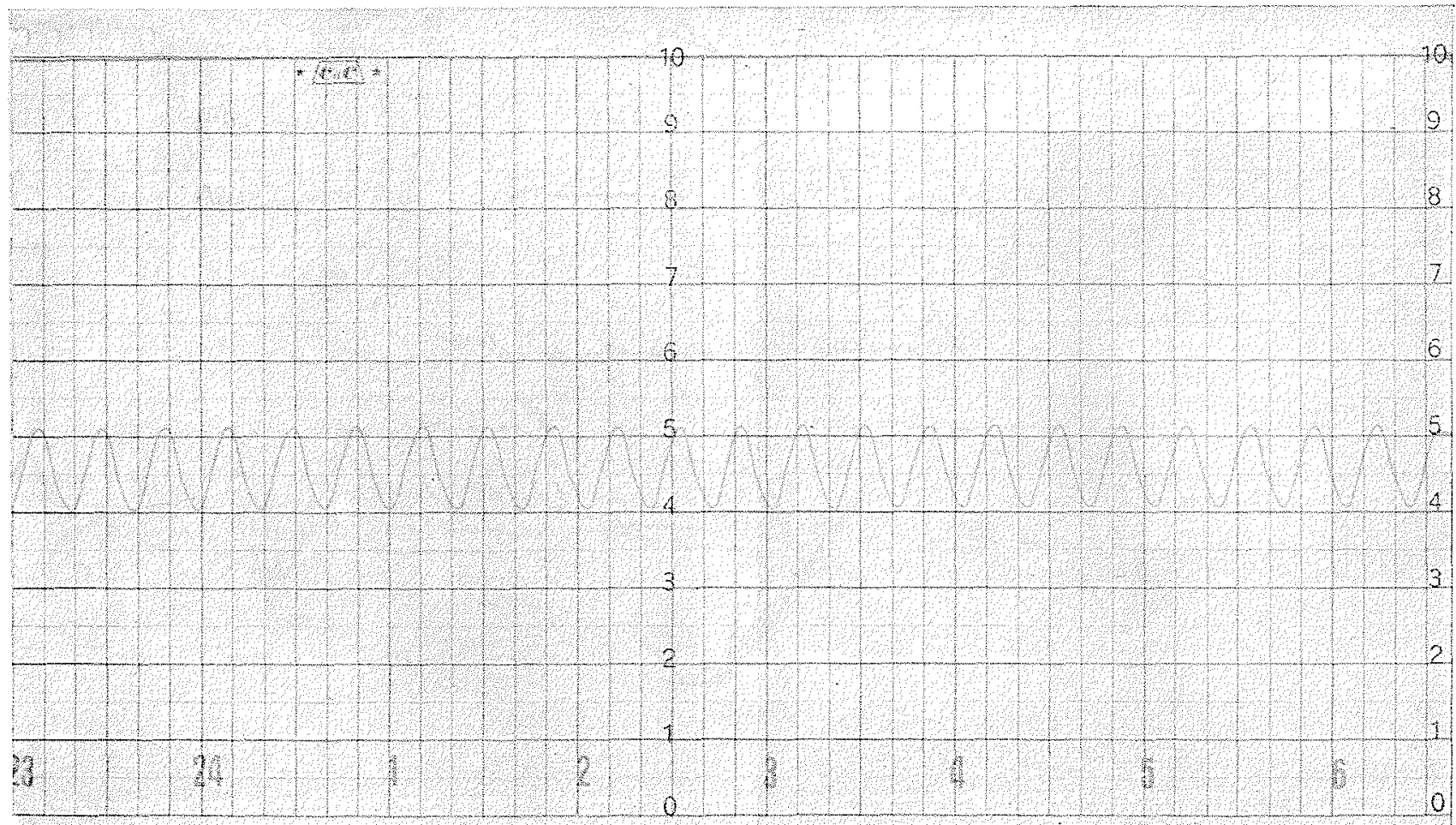

Fig. 8. 
cision de très faibles pertes de charge, rend possible la mesure industrielle de gros débits au moyen d'appareils déprimogénes à haul rendement.

Signalons enfin que, pour la réalisation d'appareils de Laboratoire à engendrer les marées sur modeles, l'emploi de pointes vibrantes nous at permis récemment de réaliser des montages particulièrement simples, précis et robustes.

En terminant, nous tenons a remercier tout particulierement M. VAcEmors, Docteur is Sciences, Ingénieur au Laboratoire, avec lequel nous avons été étroitement associé dans la conception et la réalisation de cet appareil.

\section{DISCUSSION}

W. le President remereie N. Grider et remarque qu'il est intéressant de comaître une telle méthode qui permet de mesurer les niveaux rapidement variables avec une précision de cet ordre.
Sur la demande de M. DE Mabartic, M. Grover signale (ue lappareil actuellement utilisé est réglé pour une loi d'amplitude de $5 \mathrm{~cm} / \mathrm{s}$, mais que le systeme permet de suive des variations beaucoup plus rapides.

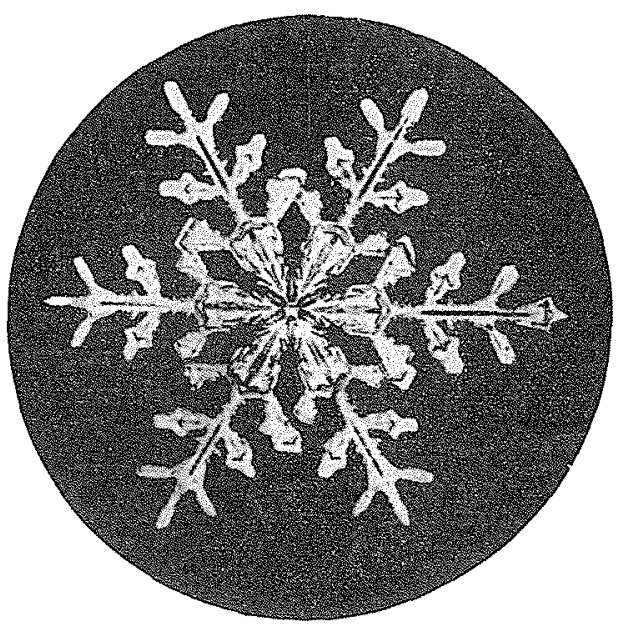

